УДК 681.3 .06

\title{
АЛГОРИТМ УСТРАНЕНИЯ СПЕКТРАЛЬНОЙ ЭКВИВАЛЕНТНОСТИ КОМПОНЕНТНЫХ БУЛЕВЫХ ФУНКЦИЙ S-БЛОКОВ КОНСТРУКЦИИ НИБЕРГ
}

\author{
СОКОЛОВ А. В., БАРАБАНОВ Н. А.
}

Одесский национальный политехнический университет, Украина, Одесса, 65044, пр. Шевченко 1

\begin{abstract}
Аннотация. Исследовано явление спектральной эквивалентности компонентных булевых функций $S$-блоков подстановки конструкции Ниберг. Предложен эффективный алгоритм устранения спектральной эквивалентности, основанный на введении в каждую компонентную булеву функцию $S$-блока подстановки случайного модификатора. Анализ сгенерированных $S$-блоков подстановки на основе предложенного алгоритма подтвердил его эффективность и показал высокое криптографическое качество $S$-блоков подстановки
\end{abstract}

Ключевые слова: S-блок подстановки; булева функция; преобразование Уолша-Адамара; спектральная эквивалентность

Основным примитивом, определяющим криптографические свойства современных блочных алгоритмов шифрования является $S$-блок подстановки. В соответствии с принципами шифрования К. Шеннона задачей $S$-блока подстановки является конфузия, т. е. обеспечение сложной и нелинейной зависимости ключа и открытого текста [1].

Типичная конструкция современного $S$-блока подстановки состоит из дешифратора, шифратора и системы взаимосвязей между ними. Например, схематическое изображение $S$-блока подстановки для трехбитного $(k=3)$ входного слова показано на рис. 1. Структура $S$-блока подстановки и его криптографические свойства полностью определяются кодирующей $Q$-последовательностью длины $N=2^{k}$, которая для приведенного примера имеет вид $\mathbf{Q}=\{5,0,4,2,6,1,7,3\}$.

В свою очередь для применения математического аппарата булевых функций для оценки

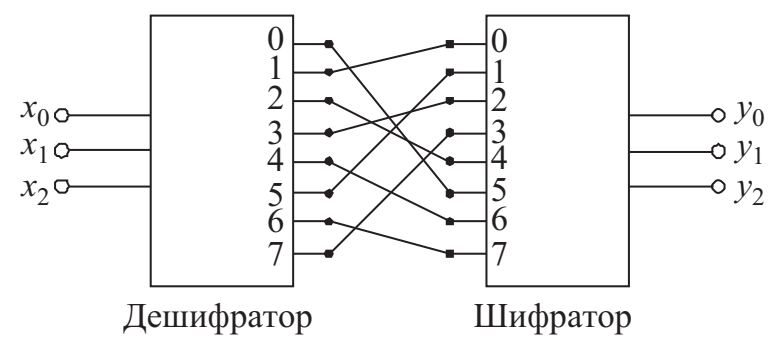

Рис. 1

криптографического качества $S$-блока подстановки кодирующая $Q$-последовательность раскладывается на множество из $k$ компонентных булевых функций $f_{1}, f_{2}, f_{3}$, представленных в виде их таблиц истинности $\mathbf{F}^{(1)}, \mathbf{F}^{(2)}, \mathbf{F}^{(3)}$. Для данного примера таблицы истинности приведены в табл. 1.

Основными критериями, предъявляемыми к современным $S$-блокам подстановки, являются высокое расстояние нелинейности, в смысле расстояния до аффинного кода, низкий уровень корреляции между входными и вы- 